\title{
Medical Images Compression and Decompression Using Neural Networks
}

\author{
Dr.B. Perumal \\ Associate Professor \\ Department of Electronics and Communication Engineering \\ Kalasalingam Academy of Research and Education \\ Krishnan koil, Tamilnadu \\ T. ChennaKeshava Reddy \\ Department of Electronics and Communication Engineering \\ Kalasalingam Academy of Research and Education \\ Krishnan koil, Tamilnadu
}

\begin{abstract}
In Emergency situations medical images of different types have to be transferred from one place to another place and the images with larger size are difficult totransfer because they are time consuming and occupies more storage. So we need image compression and decompression so that the medical images can be easily transferred and by using lossless image compression we can get the original data without any loses at receiver side. .Image compression can be made possible by using normal forward transform techniques.
\end{abstract}

But we cannot load multiple images at a time. So here we are using Support Vector Machine Neural network technique for image compression through which we can load multiple images which reduces the transmission time.

Keywords:-Lossless compression, Medical images compression, Medical images, Neural networks.

\section{INTRODUCTION}

With a lot of widespread improvement with excessive constancy rate, scientific imaging has grown to be one of the most integral fields of research. A lot of improvement has been finished to resolve quite a number of challenges incurred in scientific imaging however still, there are some challenges left unresolved. From those challenges, one of the principal challenges is the nice clinical photograph compression. Medical pictures are the images that are obtained from quite a number of photograph acquisition gadgets such as ECG (Electrocardiogram),Ultrasound(Electroencephalogram),

MRI (Magnetic Resonance Imaging), CT (computed tomography), Microscopy and PET (positron emission Tomography).Thesepictures are of high decision due to which their size becomes very large. If one needs to transmit a scientific photo over the community in order to get the opinion of a medical expert, then these scientific images can cause a heavy increase in network visitors along with high cost of storage. Thus, for having decreased storage and bandwidth requirements, compression of these

\author{
M. Harisiva \\ Department of Electronics and Communication Engineering \\ Kalasalingam Academy of Research and Education \\ Krishnan koil, Tamilnadu \\ Ch. Nikhil Reddy \\ Department of Electronics and Communication Engineering \\ Kalasalingam Academy of Research and Education \\ Krishnan koil, Tamilnadu
}

medical photographs turns into imperative . The most fundamental problem related to compression of the clinical image is formulating scientific pics with high compression ratio while retaining all the essential stats present in them alongside with the significant ability to decode the compressed clinical pictures with excessive precision. Image compression is a key innovation in the advancement of different sight and sound PC administrations and media transmission applications, for example, remotely coordinating, computerized communicate codec and video innovation, and so forth. At present, the fundamental center of picture pressure innovation comprises of three significant preparing stages: pixel changes, quantization and entropy coding. This also contributes in time saving aspects comparing to other existingtechniques.

\section{PERFORMANCEPARAMETERS}

For evaluating the quality of the resulted compressed medical images, the different parameters are calculated as shown below. Here, $\mathrm{m}, \mathrm{n}$ are representing dimensions of the image while $\mathrm{L}$ is representing the maximum grey level of original image, is representing the input image and is representing the compressedimage.

A. Mean Square ErrororMSE Rasterization. It is evaluated by the followingequation.

MSE $=$ PSNR $=20 * \log 10(255 / \operatorname{sqrt}($ MSE $))$

For having a high quality compressed image with less error, the MSE should be low.

\section{B. Bits per Pixel orBPP}

It gives the significant number of bits that are required for storing one pixel of the image. It is given by: $\mathbf{B P P}=\mathrm{S}^{\prime} /($ Total Number of Pixels $)$.

C. Compression Ratio orCR

It is calculated by the following equation:

$\mathbf{C R}=$ Suncomp $/$ Scomp

CR should be less for having a high-quality image. 


\section{Peak Signal to Noise Ratio(PSNR)}

It is calculated by the following equation

$P S N R=10 \log \left(1^{2} \quad 10 \quad / M S E \quad\right)$

The high value of PSNR signifies that the compressedimage is of high quality.

\section{PROPOSEDWORK}

In this project we are going to deal with the image compression techniques that enables in making our work much simpler in reducing the size of image and transmitting it in a very less time to the requireddestinations.
In this image compression technique we are associating with neural network, which is a very efficient and most useful technique. It also ensures that our work completes in a short time and in a effective manner.

\section{BLOCKDIAGRAM}

Image compression using neural network includes many methods and one of those methods is Support Vector Machine method of image compression which uses vectors to compress the image and it produces more accurate images when compared with other neural network techniques and through which we can load many images at a time to increase the efficiency of the process and it internally undergoes many functions such as Normalization, Segmentation.

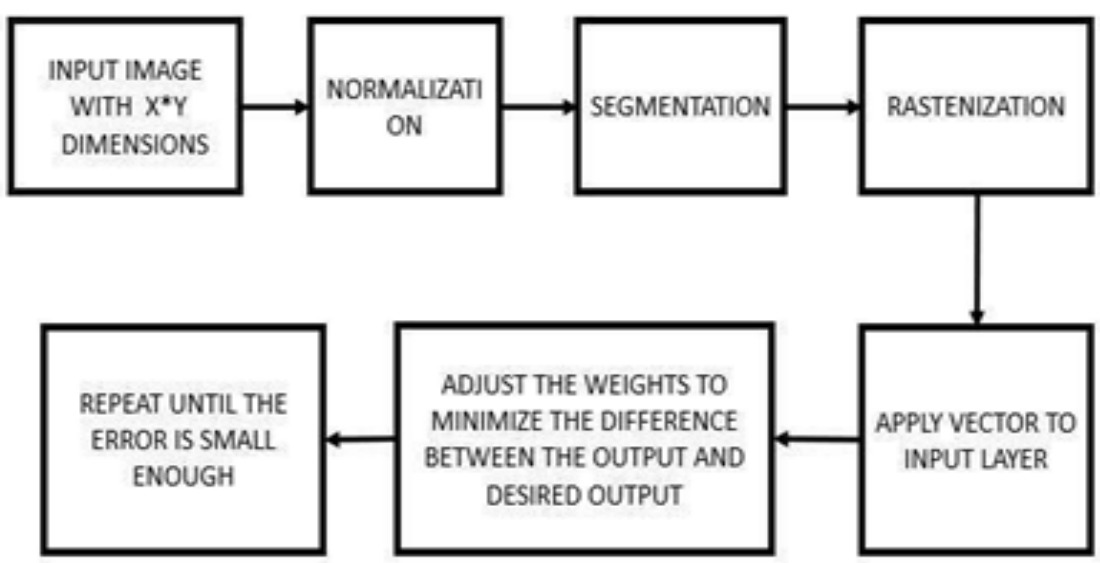

Fig 1:- Block diagram of image compression using neural network

\section{WORKING AND IMPLEMNTATION IMAGEPROCESSING}

Image Processing is a fascinating and a hot territory where everyday improvement is very baffling and has become an essential piece of claim lives. Picture preparing is the investigation, control, stockpiling, and show of graphical pictures. A picture is digitized to change over it to a structure which can be put away in a PC's memory or on some type of capacity media, for example, a hard plate. This digitization method should be possible by a scanner, or by a camcorder associated with a casing grabber board in a PC. When the picture has been digitized, it tends to be worked upon by different picture handling activities. Picture handling is a module that is fundamentally used to improve the quality and presence of high contrast pictures. It likewise upgrades the nature of the examined or faxed record, by performing tasks that expel flaws. Picture preparing tasks can be generally partitioned into three significant classifications, Image Enhancement, Image Restoration and ImageCompression.

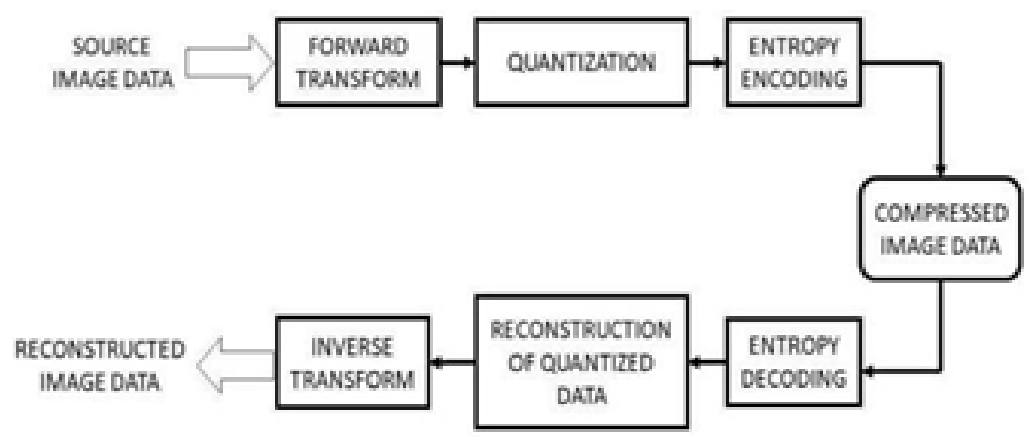

Fig 2:- Block diagram of image compression 


\section{Neural Network}

PC images are very information concentrated what's more, thus require a lot of memory for capacity. As a result, the transmission of a picture from one machine to another can be very tedious. By utilizing information pressure procedures, it is conceivable to evacuate a portion of the repetitive data contained in pictures, requiring less extra room and less time to transmit. Neural system can be utilized with the end goal of picture pressure. It is clear that neural system determines its registering power through, first its greatly equal circulation structure and second, its capacity to learn what's more, along these lines sum up. Speculation alludes to the neural arrange delivering sensible yields for inputs not experienced during training(learning). These two data preparing capacities make it workable for neural system to takecare of complexissue that are right now unmanageable.

\section{Support Vector Machine Technique}

Support vector machine (SVM) is a neural network technique which is used for image compression using this we can load multiple images at a time. And it is a supervised learning method and it is suggested by vladimirvapnik in 1992. The aim of SVM is to find the classifier to split the data and to increase the distance between classes. This classifier is called Hyper plane. The nearest points that indicate the hyper plane are called support vectors.

So we are working on a textual content classification problem. And we are refining our education data, and possibly we ha've even tried stuff out the usage of Naive Bayes. But now we are feeling assured in your dataset, and prefer to take it one step further. Enter Support Vector Machines (SVM): a quick and reliable properly with a restricted quantity of data.

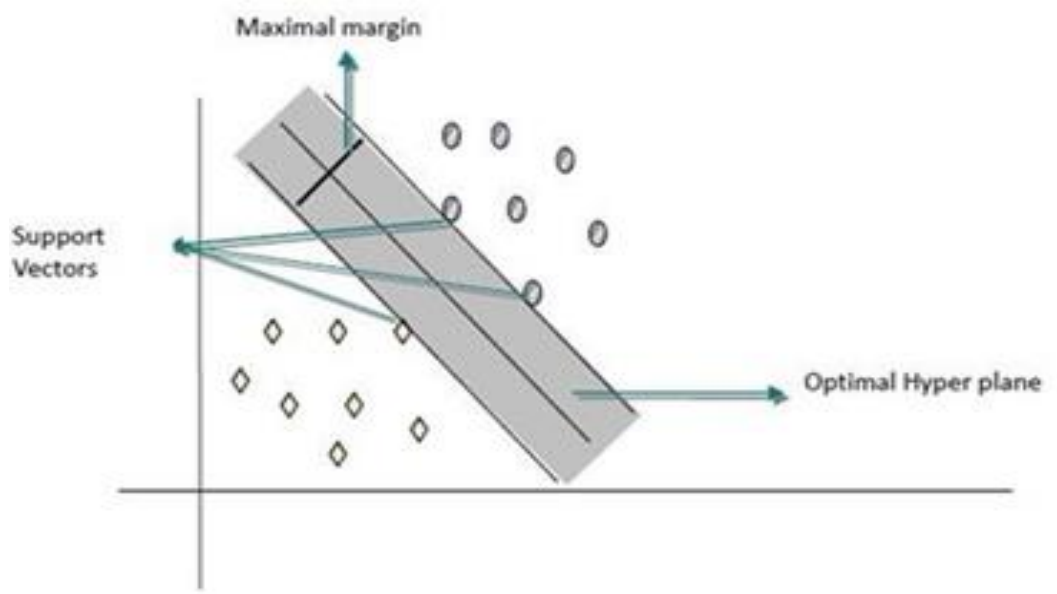

Fig 3:- Support Vector Machine

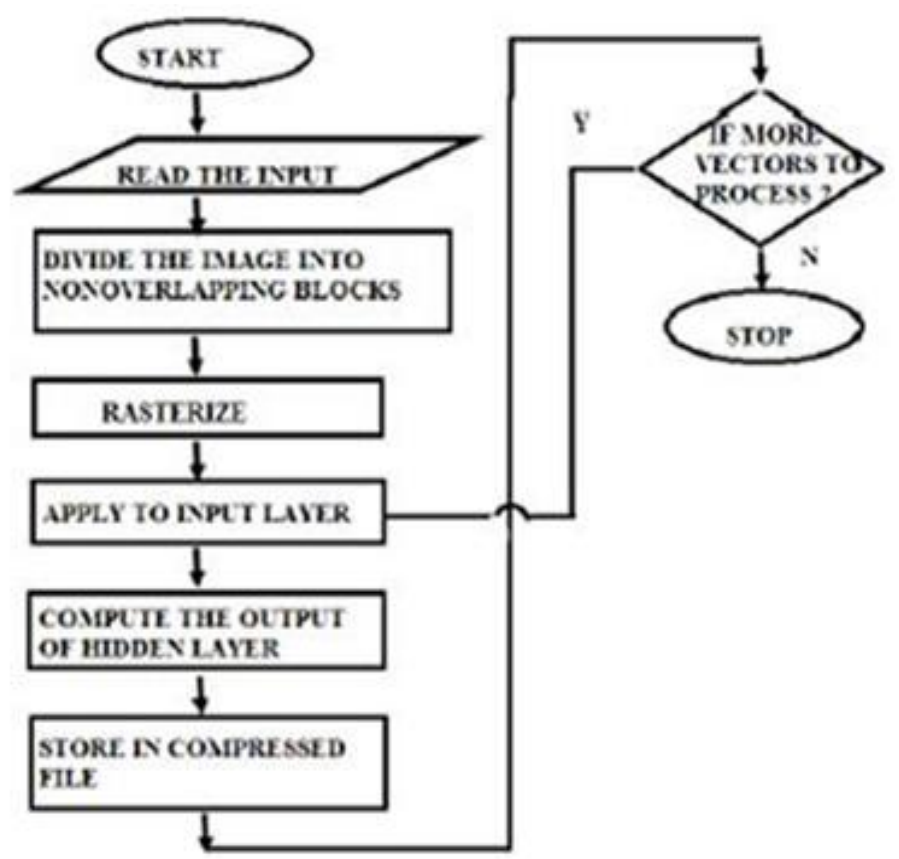

Fig 4:- work flow of image compression 
Here the work flow represents the process of image compression from loading of input image to the final compressed image. And here the work flow states that the input image is partitioned to different blocks and the esch block is rasterized that is the vector image is converted to rastre image and given as input to the input layer and passes to middle and output layers and check for any error rate. If the error rate is zero the image is successfullycompressed.

\begin{tabular}{|c|c|c|c|c|}
\hline Image size & $\begin{array}{c}\text { Compression ratio } \\
\text { value }\end{array}$ & PSNR & MSE & SNR \\
\hline $64 * 64$ & 1.11 & 30.32 & 20.32 & 25.45 \\
\hline $128 * 128$ & 1.32 & 32.42 & 14.1 & 27.13 \\
\hline $256 * 256$ & 1.51 & 37.89 & 8.32 & 32.52 \\
\hline $512 * 512$ & 1.64 & 38.88 & 3.56 & 33.17 \\
\hline
\end{tabular}

Table 1:- Final Resultant Table

\section{FUTURESCOPE}

In Future there will be a scope of compressing the larger images in faster manner. Using of this Neural network techniques are bit complex than the convolution techniques which are mostly used for image compression. Normally by compressing the image we may have some loss of data in the compressed image.

So in Future we will focus on reducing the complexity of these neural network techniques and to obtain the loss less data of the image.

\section{RESULT}

Here we have considered the medical images such as MRI, CT, PET which are mostly used for many medical purposes. And here the final result will be the original and compressed images will be same but they will be differ in size which will be illustrated below.

\section{CONCLUSION}

This paper examines work which has been proposed for effective compression of medical pictures utilizing Neural Network. In spite of the fact that these were giving critical outcomes still they have some inadequacies as far as MSE, PSNR, BPP, and CR which can be workedupon.

Additionally, medical pictures like MRI, CT, PET are the entire slide pictures which are very enormous in size ought to likewise be fused in future research.

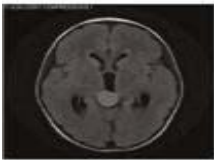

MIRI 1

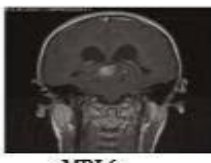

IRI6

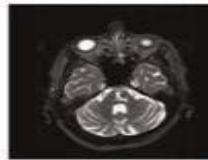

MRI 2

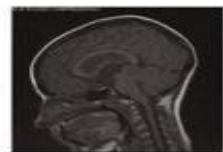

VRI 7

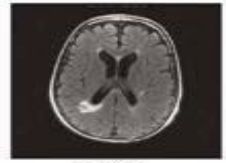

MIRI 3

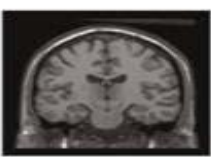

VRI8

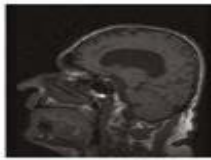

MRI 4

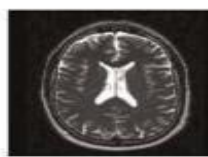

MIRI 9

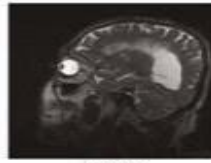

MRI 5

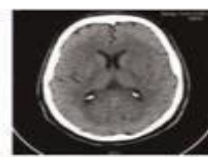

CT 10

Fig 5:- Original Medical Images

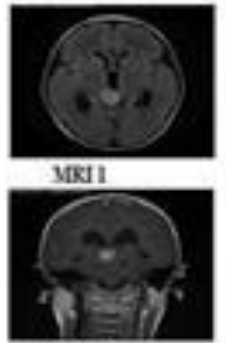

MIRI 6

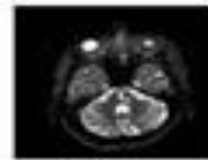

MRI2

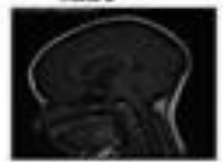

1057
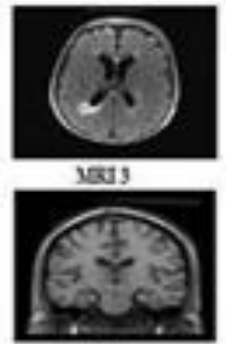

IIRI 3

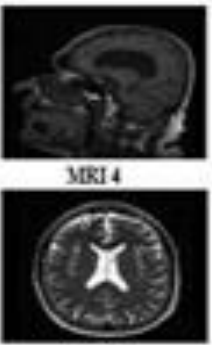

Yast
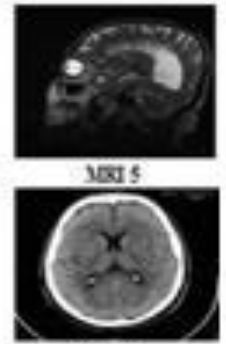

CT10

Fig 6:- SVM compression technique's resulted compressed medical image

And Here this table indicates the orginal size and the compressed size of the loaded image with the compression ratio included. 


\begin{tabular}{|c|c|c|c|c|}
\hline Name of image & Orginal size & $\begin{array}{c}\text { Compression ratio } \\
\text { [orginal image size] }\end{array}$ & $\begin{array}{c}\text { Compressed image } \\
\text { size }\end{array}$ & $\begin{array}{c}\text { Decompressed } \\
\text { image size }\end{array}$ \\
\hline Image 1 & $55.3 \mathrm{~kb}$ & 15 & $3.85 \mathrm{~kb}$ & $20.7 \mathrm{~kb}$ \\
\hline Image 2 & $33.6 \mathrm{~kb}$ & 9 & $3.35 \mathrm{~kb}$ & $17.2 \mathrm{~kb}$ \\
\hline
\end{tabular}

Table 2:- Sizes of orginal and compressed images with CR

\begin{tabular}{|c|c|c|c|c|}
\hline Image size & $\begin{array}{c}\text { Compress ion ratio } \\
\text { value[our } \\
\text { implementation] }\end{array}$ & $\begin{array}{c}\text { Compress ion ratio } \\
\text { value[ their work] }\end{array}$ & $\begin{array}{c}\text { MSE } \\
\text { values[our } \\
\text { implementation] }\end{array}$ & $\begin{array}{c}\text { MSE } \\
\text { [their } \\
\text { implementation] }\end{array}$ \\
\hline $256 * 256$ & 1.51 & 4.1 & 8.32 & 15.46 \\
\hline
\end{tabular}

Table 3:- Output values for $256 * 256$ image

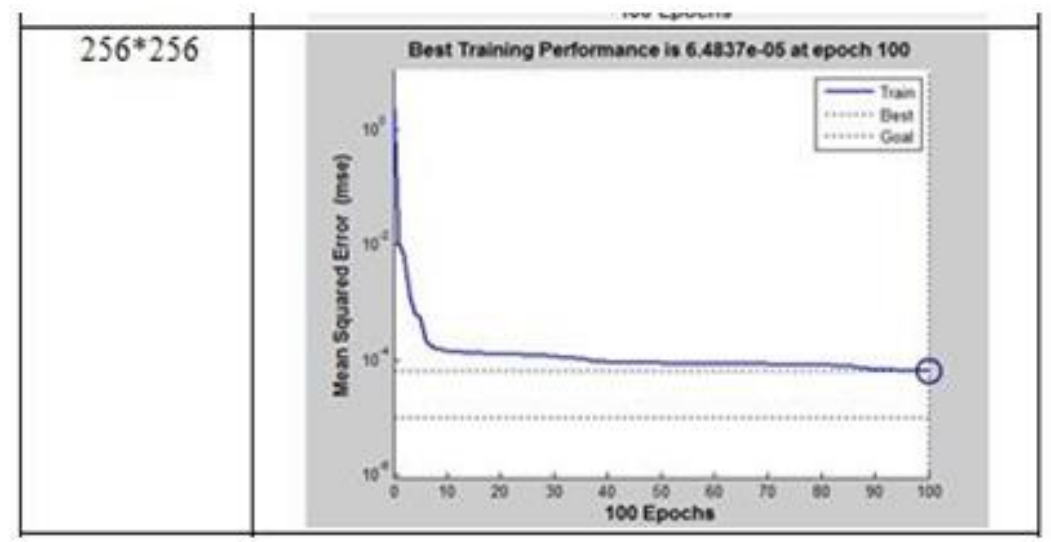

Fig 7:- Performance plot for $256 * 256$ image

\section{REFERENCES}

[1]. W. Hwang, C.F. Chine, K.J. Li, "Scalable medical data compression and transmission using wavelet transform for telemedicine applications," IEEE Trans. Inf. Tech. Biomed., vol. 7, no. 1, pp. 54-63,2019

[2]. M. Dahiya, S. Sukhija, and H. Singh, "Image encryption using quad phase masks in fractional Fourier domain and case study," in Proc. IEEE International Advance Computing Conference (IACC), 2018.

[3]. V. Sanchez, R. Abugharbieh, and P. Nasiopoulos, "Symmetry-based scalable lossless compression of 3D medical image data," IEEE Trans. Med. Imaging, vol. 28, no. 7, pp. 1062-1072,2018.

[4]. S.G. Chang and G.S. Yovanof, "A Simple BlockBased Lossless Image CompressionScheme," in Proc. Thirtieth Asilomar Conference on Signals, Systems, and Computer, 2017.

[5]. Y.T. Chen, D.C. Tseng, and P.C. Chang, "Waveletbased Medical Image Compression withAdaptive Prediction," in Proc. International Symposium on Intelligent Signal Processing and Communication Systems, 2017.

[6]. Y.T. Chen, D.C. Tseng, and P.C. Chang, "Waveletbased Medical Image Compression withAdaptive Prediction," in Proc. International Symposium on Intelligent Signal Processing and Communication Systems, 2017.
[7]. R. Matsuoka, M. Sone, K. Fukue, K. Cho, and H. Shimoda, "Quantitative Analysis of Image Quality Of Lossy Compression Images," in Proc. International Society for Photogrammetry and Remote Sensing,2016.

[8]. G.K. Wallace, "The JPEG Still Picture Compression Standard," IEEE Transactions on Consumer Electronics, vol. 38, no. 1, pp. 17-34,2016.

[9]. S. C. V. Yadav, Ms. S. R.Gaikwad, Ms. N. N. Jakhalekar, Mr. V. A. Patil" Image Compression and Reconstruction Using Artificial Neural Network", Vol 03, Jan Issue:02|Feb-2016. 